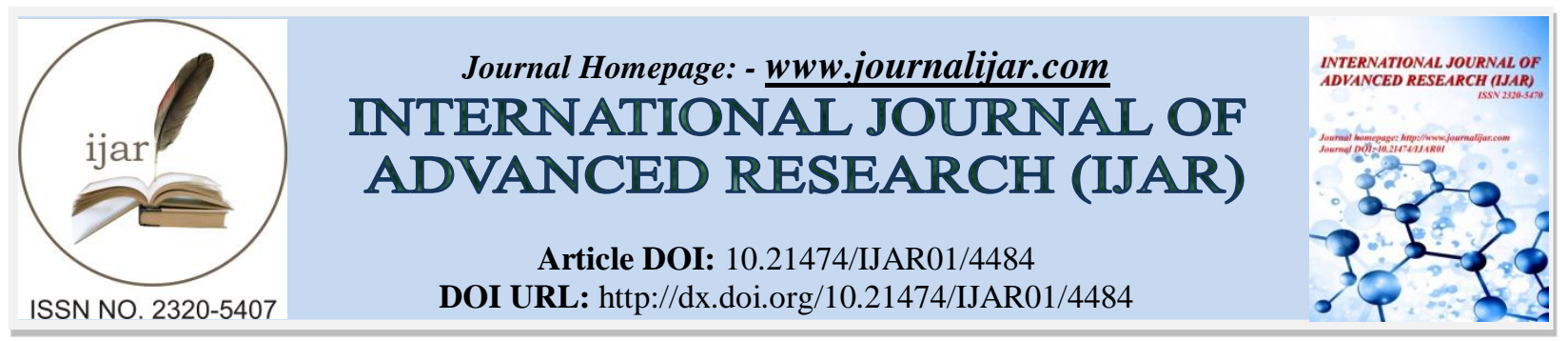

RESEARCH ARTICLE

\title{
NANOTECHNOLOGY IN DENTISTRY-A REVIEW.
}

Dr. A. Vasanthakumari ${ }^{1}$, Dr. Vivek Reddy ${ }^{2}$, Dr. K. Vivek ${ }^{2}$ and Dr. D. Bhavani Pratima ${ }^{3}$.

1. Prof \& Head, Department of Pedodontics and Preventive Dentistry, Adhiparasakthi Dental College \& Hospital, Melmaruvathur, Tamilnadu, India-603319.

2. Senior lecturers, Department of Pedodontics and Preventive Dentistry, Adhiparasakthi Dental College \& Hospital, Melmaruvathur, Tamilnadu, India-603319.

3. Lecturer, Department of Pedodontics and Preventive Dentistry, Adhiparasakthi Dental College \& Hospital, Melmaruvathur, Tamilnadu, India-603319.

\section{Manuscript Info}

Manuscript History

Received: 14 April 2017

Final Accepted: 16 May 2017

Published: June 2017

\section{Key words:-}

Nanodentistry, Nanomaterials, Nanotechnology.

\section{Abstract}

Man's quest to create new technology and materials which are better and more efficient led to the introduction of nanotechnology that deals with structures ranging in the size of 100 nanometers or smaller in at least one dimension and developing materials or devices within that size. The basic idea of nanotechnology is to employ individual atoms and molecules to construct functional structures. Nanotechnology has revolutionized all fields from healthcare to engineering into new archetype beyond traditional and dentistry is no exception. This review provides the role of nanomaterials and their potential to be used in the diagnosis and management of oral diseases.

Copy Right, IJAR, 2017,. All rights reserved.

\section{Introduction:-}

Rationalizing has become a new trend in the world of science and technology. Nanotechnology has ascended one of the most favourable technologies and one which change the application of materials in different fields. The quality of dental biomaterials has been improved by the emergence of nanotechnology. This technology manufactures materials with much better properties or by improving the properties of existing materials. The science of nanotechnology has become the most popular area of research, currently covering a broad range of applications in dentistry. ${ }^{1}$ Nanotechnology is the engineering of functional system at the molecular scale. It deals with structures ranging in the size of 100 nanometers or smaller in at least one dimension and developing materials or devices within that size. The basic idea of nanotechnology is to employ individual atoms and molecules to construct functional structures. It gives us an understanding of how structures are made at a fundamental level and how their molecular arrangements can be altered to alter the macroscopic properties of a material ${ }^{2}$.

\section{History:-}

The term nanotechnology is derived from the Greek word 'nanos' meaning dwarf. The Nobel Prize winning physician Richard.P.Fenyman, during his 1959, plenty of room at the Bottom speech to the American Physical Society, had first projected this dimensions of discoveries at a billionth meter scale. The term nanotechnology was introduced by Norio Taniguchi in 1974, when he referred to a 'production technique to get extra high accuracy and ultrafine dimensions'. Later in 1986, K.Eric Drexler contributed to its development by introducing the concept of molecular nanotechnology in his 1986 publication, Engines of creation, the coming era of nanotechnology.

Corresponding Author:- Dr. A. Vasanthakumari.

Address:- Prof \& Head, Department of Pedodontics and Preventive Dentistry, Adhiparasakthi Dental 
Applications in the field started in the 1980's with the invention of scanning tunnelling microscope and the discovery of carbon nanotube and fillers. However, major initiatives began at the beginning of this century, thus ushering in the era of nanotechnology ${ }^{3}$.

\section{Fundamental Concepts Of Nanotechnology:-}

Nanotechnology is the manipulation of matter on the molecular and atomic levels. It is measured in the billionths of meters or nanometer, roughly the size of two or three atoms. One nanometer is one billionth $10^{-9}$ of a meter. Nanomaterials are materials with components less than $100 \mathrm{~nm}$ in atleast one dimension, including the cluster of atoms, grains less than $100 \mathrm{~nm}$ in size, fibres less than $100 \mathrm{~nm}$ in diameter, films less than $100 \mathrm{~nm}$ in thickness. Nanoholes and composites that are a combination of these. To put that scale in another context, the comparative size of a nanometer to a meter in the same as that of a marble to the size of the earth. A unique aspect of nanotechnology is the vastly increased ratio of surface area to volume present in many nanoscale materials which opens new possibilities $^{4}$

Nano Materials And Nano Devices:-

Siegel has classified nanomaterials as zero dimensional. If present in one dimension is called as sheet, if in two dimensions are nanowires and nanotubes, and if present in three dimensions are called as quantum dots.

\section{The various Nanostructures include:-}

- Nanopores

- Nanoparticles

- Nanotubes

- Nanorods

- Nanospheres

- Nano fibres

- Nano shells

- Dendrimers and dendritic copolymers.

Inorganic nanoparticles either currently in use or under development include:- 5

- Semiconductor nanoparticles

- Metal nanoparticles

- Metaloxide nanoparticles

- Silica nanoparticles

- Polyoxometalates

- Gold nanocrystals

Advent Of Nano Technology:-

The invention of scanning tunneling microscope (STM) and atomic force microscope are the achievements that developed nanotechnology through the scientific method rather than a conceptual one. Owing to the invention of scanning tunneling microscope, by Binning and Rohrer in 1981, it was for the first time that the individual atoms could be easily identified. Some of the limitations of this microscopy where eliminated through the second invention in the series, the atomic force microscope, which could image, non-conducting materials such as organic molecules. This invention was integral for the study of carbon buckyballs, discovered at Rice University in 1986 and carbon nanotubes few years later ${ }^{6}$.

\section{Creation Of Nanoproducts:-}

A number of nanotechnology approaches are begin used for a range of practical applications in dentistry. There are three approaches used in nano technology for creating smaller or better materials and use of smaller constituent into more complex assembling ${ }^{7}$.

\section{Top down Approach:-}

It is based on solid- state processing of materials. Typical examples of top down processes are milling machining and lithography. The top down approaches such as chemical vapor deposition (CVD) monolithic processing, wet and plasma etching are used to fabricate functional structures at micro and nano scales. These approaches are 
successfully used in electronic industry and for coatings of medical implants and start using CVD technology to enhance blood flow and biocompatibility.

\section{Bottom- up Approach:-}

It entangles the fabrication of materials via edifice up particles by harvesting atomic elements. Bottom up processing is based on extremely organized chemical synthesis and growth of materials. The best example of this approach is present in nature, eg: repairing of cells, tissues or organ systems and proteins as well.

\section{Functional Approach:-}

In this approach, components of a desired functionality are developed without regard to their ascending or descending order of assembly and in addition to this, Rice University follows three additional techniques.

\section{Wet Nanotechnology:-}

All biological systems are having water as a content like genetic materials, membranes, enzymes and nano-sized cellular components exist mainly in water environment.

\section{Dry Nanotechnology:}

It is applicable on physical and surface materials which may be organic or inorganic for example carbon and silicon. This technology is derived from surface science and physical chemistry, it focuses on fabrication of structure.

\section{Computational Nanotechnology:-}

It permits the structuring as well as stimulation of complex nanometric structures .It is multidisciplinary owing to its application in diagnostic medicine, dry delivery systems, genetic engineering, surgeries, cosmetic dentistry and many more ${ }^{8}$.

\section{Nanomedicine And Nanodentistry:-}

Nanomedicine is the science and technology of diagnosing, treating and preventing disease and traumatic injury relieving pain and presenting and improving human health through the use of nanoscale structured materials, biotechnology and genetic engineering, eventually complex molecular machine systems and nanorobots. Nanodentistry will make possible the maintenance of near-perfect oral health through the use of nanomaterials, diagnosis, biotechnology including tissue engineering and nanorobotics 9 .

\section{Nanodentistry:-}

There has been remarkable research on nanomaterials in recent years, which has moved it from theoretical foundation to clinical practice. Currently, there is a wide range of nanomaterials application in different specialities of dentistry ${ }^{10}$.

\section{Application In Dentistry:-}

- Top Down Approach

- Bottom-Up Approach

\begin{tabular}{|l|l|}
\hline Nanocomposites and \\
Nanoclusters. \\
\cline { 2 - 2 } $\begin{array}{l}\text { Nano-light curable glass } \\
\text { ionomer cements }\end{array}$ \\
\begin{tabular}{|l|} 
Nano-impression materials. \\
Nanoparticles coating in \\
Dental implants
\end{tabular} \\
\hline $\begin{array}{l}\text { Nanobased bone } \\
\text { replacement cements }\end{array}$ \\
\hline Nanoencapsulation. \\
\hline Nanoneedles. \\
\hline \\
\hline Local anesthesia \\
\hline
\end{tabular}




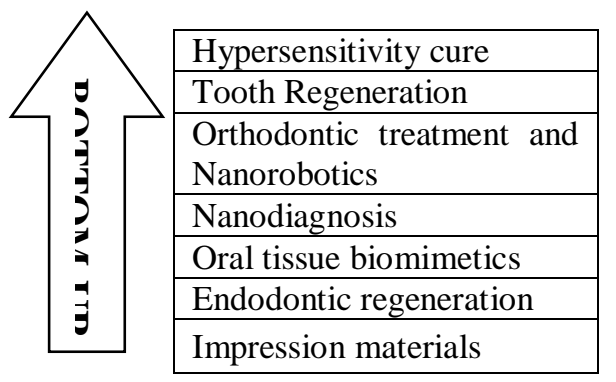

Current Application Of Nanotechnology In Dentistry-:

The field of dentistry is receiving unprecedented support from the biological sector in the form of novel innovation that include improvised diagnostic aid and treatment devices. Current dental research involves progressive ingress into the diagnostic, reconstructive, regenerative, restorative and space rehabilitative and preventive domain ${ }^{11}$.

\section{Application Of Nanotechnology In Diagnostic Dentistry:-}

Dental caries and periodontal disease are the most common affecting the human race. Methods to prevent and combat them have been devised, discussed and implemented since ancient times .However there is a constant need for improved tools and techniques. Nanotechnology with its ever increasing scope, provides dental research new opportunities for progress. ${ }^{12}$

\section{Biofilm:-}

Biofilm are considered the root cause of most dental and periodontal diseases. Technology employing nanosized quantum dots based on immunofluorescence enables the labelling of specific bacteria, which cases their identification and removal. The contribution of modern technology in the field of oral microbiology started with the detection of cultivable as well as uncultivable bacteria by examining bacterial 16S RNA and DNA. Nanotechnology has been used to study the dynamics of demineralization/remineralization process in dental caries by using tools such as atomic force microscopy(AFM) which detect bacteria induced demineralization at an ultrasensitive level ${ }^{13}$.

Another nanotechnology application used so far is $\mathrm{O}^{16} / \mathrm{O}^{18}$ reverse proteolytic labelling to determine the effect of biofilm cuture on the cell envelope proteome of oral pathogen. Porphyromonas gingivalis species which is linked to chronic periodontitis. New silver nanotechnology chemistry has proven to be effective against biofilms. It has a high affinity for negatively charged side groups on biological molecules such as sulphydryl, carboxyl, phosphate and other charged groups distributed throughout microbial cells. Recent studies show that ionic plasma disposition silver anti-microbial nanotechnology is effective against pathogens associated with biofilm including E.colisp, S.pnuemoniae sp, S.pneumoniae, S.aureus, A.niger ${ }^{14}$

\section{Detection Of Salivary Biomarkers:-}

Highly sensitive diagnostic techniques involving better revelations of autoantibodies, dysplastic cells, and salivary biomarkers are required for diagnosis and early treatment. Cost effective technological advancements on this front will help promote a wide spread implementations of salivary diagnostis. The oral fluid Nano sensor test (OFNASET), which enables point of care detection of salivary proteomic biomarkers and nucleic acids specific for oral cancer, including 4mRNA biomarkers (SAT,ODZ,IL-8, and IL-1beta) and 2 proteomic biomarkers (Thioredoxin and $\mathrm{IL}-8)^{15}$.

\section{Application Of Nanotechnology In Restorative Dentistry:-}

There has been a drastic evolution in recent years for restorative materials, particularly in tooth color materials.

\section{Local Anaesthesia: -}

Nanodentistry, a colloidal suspension containing millions of active analgesic micron-size dental robots will be instilled on the patient's gingiva. After contacting the surface of crown or mucosa, the ambulating nanorobots reach with the pulp via the gingival sulcus, lamina propria and dentinal tubules guided by chemical gradient, temperature differentials all under the control of dentists, will help of on-board nanocomputer. Once installed in the pulp, the analgesic dental robots may be commanded by the dentist to shut down all the sensitivity in any particular tooth that requires the treatment. This technique avoids most of the side effects and complications ${ }^{16}$. 


\section{Hypersensitivty Cure:-}

Dental nanorobots could selectively and precisely occlude selected tubules in minutes using native biological materials, offering patients, a quick and permanent cure.

\section{Dental Biomimetics:-}

The central theme of biomimetics is the way to mimic nature's already efficient use of nanotechnology which involves interaction between amelogenin and the formation and directional orientation of Hydroxyapatite crystals to compose enamel. The results may resemble processes that occur in actual enamel formation.

\section{Endodontic Regeneration:-}

Current treatment modalities of regenerative approaches will result in the replacement of diseased or necrotic pulp with healthy pulp tissues to revitalize teeth. The use of nanostructural and functionalized multi-layered films containing a-MSH as a new active nanobiomaterial for endodontic regeneration ${ }^{17}$.

\section{Nanocomposite:-}

The current clinical utility of nanotechnology's most tangible contribution to dentistry is the nanocomposites which are primarily in terms of smoothness, polishability and precision of shade characterization, flexural strength and microhardness similar to or more than conventional RBCs. Ormocers with nanoparticle fillers and widely used in nanocomposite restorative systems. Modifying ormocers with organic moieties such as methacrylate-substituted $\mathrm{ZrO}_{2}$ or $\mathrm{SiO}_{2}$ organosol nanoparticles was to improve the mechanical properties and biocompatiblity of RBCs.

\section{Nanolight Curing Glass Ionomer Restorative:-}

By using bonded nanofillers and nanoclusters fillers, manufacturers have begun to incorporate nanoscale structuring to produce nanoionomers in an effort to make their surface finish more closely approximate that of a hybrid composite and lesser wear.

\section{Pit And Fissure Sealant:-}

The nanofissure sealant results in outstanding wear resistance and a reduced shrinkage, feasible sealing ability, hydrophilic material and easy to place. Due to high fluoride release it may lead to remineralization.

\section{Nanoadhesive:-}

They are nanosolutions which produce unique and dispersible nanoparticles which prevent agglomerations and had longer shelf life, high stress absorption, fluoride release and durable marginal seal.

\section{Nanoimpression Material:-}

Nanofillers have been added to polyvinyl siloxane material to enhance its properties. It has better flow, fewer voids and enhanced detail precision.

\section{Bone Replacement Materials:-}

Hydroxyapatite nanoparticles used to treat bone defects are ostim, vitosso, Nanosstm. These are used in maxillofacial injuries requiring bone graft, cleft patient, endodontic surgeries and osseous defects in periodontal surgeries ${ }^{18}$.

\section{Application Of Nanotechnolgy In Periodontics:-}

Nanorobotics is the technology of creating machines or robots used in many areas in the field of periodontics.

Nanorobots incorporated in mouthwash could identify and destroy pathogenic bacteria leaving behind harmless oral flora to flourish in the oral ecosystem. Continuous debridement of supra and subgingival calculus would be done by nanorobots incorporated in dentrifice. They provide continuous barrier to halitosis.

\section{Nano Toothpaste:-}

Nano whitening tooth paste that contain synthesized hydroxyapatite, a key component of tooth enamel as a nanosized crystals. It has been proven to freshen breath as well as whiten teeth. 


\section{Periodontal Tissue Engineering:-}

Nanotechnology has lot of potential to produce non biologic self-assembling systems for tissue engineering purposes. It is possible to create polymer scaffolds in the future for cell seeding, growth factor delivery and tissue engineering via nanodevices implanted to the sites of tissue damage.

\section{Dentinal Hypersensitivity:-}

Reconstructive dental nanorobots, using native biological materials could selectively and precisely occlude specific tubules within minute offering patients, a quick and permanent cure.

\section{Tooth Repair:-}

The use of nanotechnology to simulate the natural bio-mineralisation process to create the hardest tissue in the body, the enamel by using highly organized micro architectural units of nano-rod like calcium hydroxyapatite crystals arranged parallel to eachother. ${ }^{19}$

Application Of Nanotechnology In Orthodontics:-

Recently the unprecedented progress in treatment, modalities have been witnessed in the field of orthodontics, the self-ligating brackets, customized treatment techniques and invisalign to name the few.

\section{Acid Etching Technique:-}

It was introduced in the mid 1950's and became universally accepted in the 1960's.It took more than a decade for this practice to become standard procedure in orthodontics in a large scale by nanotechnology.

\section{Tooth Repositioning:-}

Nanoparticles have recently channelized their way in orthodontics in various modalities like bracket less tooth repositioning, nano-coated orthodontic wires to reduce friction and also by alveolar tissue augmentation.

Orthodontic nanorobots could directly manipulate the periodontal tissue allowing rapid and painless tooth straightening, rotating and vertical repositioning within minutes to hours ${ }^{20}$.

\section{Application Of Nanotechnology In Regenerative Dentistry:-}

Nanotechnology playing major role in bone grafting, guided tissue regeneration, tissue engineering nerve regeneration and pulp regeneration. ${ }^{21}$.

\section{Application Of Nanotechnology In Rehabilitative Dentistry:-}

The introduction of dental implants has revolutionized the rehabilitative dental procedures. Implants coated with nanostructured titanium, hydroxyapatite or pharmacological agents such as bisphosphonates may induce and promote cellular differentiation and proliferation. Recently trials have also been conducted to introduce antimiocrobial bioactive implant surfaces. Recently three nano-structured implant coating are developed.

$>$ Nanostructured diamond

$>$ Nanostructured processing applied to hydroxyapatite coatings.

$>$ Nanostructured metalloceramic coatings.

$>$ Nanostructured ceramics, carbon fibres, polymers, metal and composites enhance osteoblast adhesion and calcium / phosphate mineral deposition ${ }^{22}$.

\section{Challenges Faced By Nanodentistry:-}

- Precise positioning and assembly of molecular scale part.

- Economical nanorobot mass production technique.

- Biocompatibility

- Simultaneous coordination of activities of large numbers of independent micron-scale robots.

- Social issues of public acceptance, ethics, regulation and human safety.

\section{Problems For Research In Nanotechnology:-}

- Painful, slow strategic decision

- Problem of retention of private enterprises.

- Sub-optimal funding 
- Problem of retention of trained man power

Future:-

Nanotechnology is foreseen to change health care in a fundamental way.

- Novel methods for disease diagnosis and prevention

- Therapeutic selection tailored to the patient's profile.

- Drug delivery and fine therapy.

\section{Conclusion:-}

Nanodentistry will give a new vision to comprehensive oral health care, as new trends of oral health have been changing to more preventive intervention than a curative and restorative procedure. It has a strong potential to revolutionize dentistry as to diagnose and treat dental diseases in future. Nanodentistry still focus many significant challenges in realizing its tremendous potential. There are larger social issues of public acceptance, ethics, regulations and human safety that must be addressed before molecular nanotechnology can enter the modern medical armamentarium ${ }^{23}$.

\section{References:-}

1. Mitra S, Holmes B. An application of nanotechnology in advanced dental material. J.Am Dent Assoc 2003; 134(10):1382-1390.

2. Kumar SR, Vijayalakshmi R. Nanotechnology in dentistry. Ind J Dent 2006;17(2): 62-65

3. Freitas RA. Nanodentistry. J.Am.Dent. Assoc 2000;131(11):1559-1565

4. Verma SK et al. A critical review of the implication of nanotechnology in modern dental practice .National J of oral maxillofacial surgery .2010:1:41-44.

5. Chen MH. Update on Dental Nanocomposites. J Dent Res 2010; 89(6): 549-560.

6. Bhardwaj SB, Mehta M, Gauba K. Nanotechnology: Role in dental biofilms. Indian J Dent Res 2009:20(4):511-513.

7. Paul W, Sharma CP. Nanoceramic Matrices: Biomedical Applications. Am J BiochemBiotech 2006; 2(2):4148.

8. Rybachuk AV, Chekman IS, Nebesna NY. Nanotechnology and nanoparticles in dentistry. Pharmacology and Pharmaceutics 2009; 1: 18-21.

9. Satyanarayana \& Rai. Nanotechnology - The future. Journal of Interdisciplinary Dentistry. 2011; 1(2); 93100.

10. Freitas R. Nanodentistry. JADA 2000: 131: 1559-65.

11. Kanaparthy R, Kanaparthy A. The changing face of dentistry nanotechnology. International $\mathbf{J}$ of Nanomedicine. 2011; 6: 2799-2804.

12. Slavkin HC. Entering the era of molecular dentistry.J.Am.Dent.Asso 1999; 130:413-417.

13. Jhaver HM, Balaji. Nanotechnology: The future of Dentistry.2005; 5; 15-17.

14. Patil M, Mehta DS, Guvva S. Future impact of nanotechnology on medicine and dentistry. J.of.Ind.Soc. Periodontology. 2008; 12: 34-40

15. Jhaveri HM, Balaji. Nanotechnology: The future of dentistry. Journal of Indian Society of Periodontology.2005; 5:15-17.

16. Ozak ST, Ozak P. Nanpotechnology \& dentistry.Euro.J.Of.Dent.2013; 7:145-151.

17. Bharadwaj A, Misuriya A. Nanotechnology in dentistry: present and future. J. Of .Int. Oral Health.2014;6:121-126

18. Kong LX, peng.Z, Li SD Bartold PM. Nanotechnology and its role in management of periodontal diseases. Periodontology.2006;40:184-19

19. Neetha J, shetty P, Swati k, David. Nano robots: Future in dentistry. The Saudi.Dent.Journal.2013;25:49-52

20. Chandki R, Kala M, Kumar NK, Brigit B, Banthia P, Banthia R. Nanodentistry: Exploring the beauty of miniature. J.of.clinic.Exp.Dent.2012;4:119-124

21. Satyanarayana T. Rai R. Nanotechnology, The future. J.Of.Interdis.Dent 2012;1:93-100

22. Fakruddin Md, Hossain Z, Afroz H. Prospects and applications of nanobiotechnology: A medical perspective .J.Of Neurobio.

23. Sharma.S, cross SE, Hsuch c, Wali RP, Stiez AZ. Gimzewski JK. Nanocharacterization in dentistry. Int.J.Of.Mol.Sci.2010;11:2523-2545 